\title{
THE USE OF A DRIVING SIMULATOR TO ASSESS SENIOR DRIVER PERFORMANCE: INCREASING SITUATIONAL AWARENESS THROUGH POST-DRIVE ONE-ON-ONE ADVISEMENT
}

\author{
Matthew R. E. Romoser ${ }^{1}$, Donald L. Fisher ${ }^{1}$, \\ Ronald Mourant ${ }^{2}$, Jerry Wachtel ${ }^{3}$, Konstantin Sizov ${ }^{4}$ \\ ${ }^{1}$ Human Performance Laboratory \\ Mechanical \& Industrial Engineering Department \\ University of Massachusetts, Amherst \\ Amherst, Massachusetts, USA \\ E-mail: mromoser@ecs.umass.edu \\ E-mail: fisher@ecs.umass.edu \\ ${ }^{2}$ Virtual Environments Laboratory \\ Mechanical, Industrial and Manufacturing Engineering Department \\ Northeastern University \\ Boston, Massachusetts, USA \\ E-mail: mourant@.coe.neu.edu \\ ${ }^{3}$ The Veridian Group, Inc. \\ Berkeley, California, USA \\ E-mail: jerwachtel@aol.com \\ ${ }^{4}$ Drive Square LLC. \\ Newton Centre, Massachusetts, USA \\ E-mail:ksizov@drivesquare.com
}

\begin{abstract}
Summary: Older drivers are over-represented in angled impact crashes and experience a higher fatality rate than their younger counterparts. Due to the gradual deterioration of the senses, diminished cognitive processing capabilities and decreased mobility and flexibility, it is more difficult for older drivers to gather and process information about their environment. This can lead older drivers to incorrectly perceive their driving environment as safe, when in reality it is not. The current study investigates whether post-drive feedback following a simulated drive can effectively change older drivers' attitudes about their own driving ability and influence them to incorporate additional compensatory behaviors into their day-to-day driving.
\end{abstract}

\section{INTRODUCTION}

The literature on how age impacts driving ability contains the now familiar U-shaped curve describing the number of accidents per vehicle mile traveled (VMT) versus age (the actual units and quantity of distance reported in the literature vary). From licensing age - usually 16 - to age 25, accidents per VMT decrease. After age 25, accidents per VMT reach a type of steady state and remain about the same until around age 70-75, when the accident rate per VMT begins to increase again and eventually begins to approach the same rate as relatively inexperienced drivers under the age of 18 . This relationship has proven to be repeatable all over the world, 
regardless of where the data has been collected (Bryer, 2000; Cook et al., 2000; Evans, 1987; Graca, 1986; Hakamies-Blomqvist, 1996; Lyman et al., 2002; Ryan et al., 1998). Older drivers are also more likely to be killed in auto accidents than their younger counterparts. This is especially true after age 85 when, according to the IIHS (2003), fatalities increase from 1.7 fatalities per 100 million miles driven for the 65 to 70 age range to nearly 13.4 fatalities per 100 million miles for drivers 85 and over. According to data presented by Bryer (2000), fatality rates per 100,000 licensed drivers are highest for drivers 85 and older (30.71), followed by drivers between 16 and 19 years old (22.08).

Why does the number of accidents per VMT begin to climb again after age 75 ? Presumably, most drivers over 75 years of age have been driving most of their adult lives, so with the exception of very few cases, lack of experience can usually be eliminated as a contributing factor to increased accident rates. It then becomes necessary to consider other potential contributing factors. Age-related cognitive slowing, ocular motor difficulties, sensory channel processing, distractibility, visual quality deterioration, diminished useful field of view (UFOV) and dementia, as well as advancing physical limitations affecting posture, flexibility, movement speed, and psychomotor control, can all combine to make it more difficult for an elderly driver to collect and process the information or cues present in a dynamic driving environment (Ball et al., 1993; Ball \& Owsley, 2000; DeRaedt \& Ponjaert-Kristoffersen, 2000; Fozard, 2000; HakamiesBlomqvist, 1996; Klavora \& Heslegrave, 2002).

Being able to detect and perceive cues in a dynamic driving environment, assigning a meaning to those cues, and projecting the future state of the driving environment based upon those cues is critical for all drivers to be able to react and plan their actions accordingly while driving (Carpenter et al., 2004; Moulaoua et al., 2004; Smither et al., 2004). Being able to do these three things successfully is the basic tenet behind cognitive models of situational awareness. According to Endsley (2000), situational awareness (SA) can be thought of as having three levels. Level $1 \mathrm{SA}$ (perception) involves the ability to perceive elements in the current environment or situation. She argues that without a basic perception of important information, the odds of forming an incorrect picture of the situation increase dramatically. In older adults the visual, cognitive, psychomotor and physical problems listed above may negatively impact Level 1 SA because they can all affect how well a driver can scan his environment. Level 2 SA (comprehension) involves the integration of multiple pieces of information, determining their relevance to the goals of the situation. In older adults, aspects like cognitive slowing, diminished UFOV (Ball et al., 1988), or decreased short-term memory span might all impact Level 2 SA, since processing information in a quickly changing environment is critical to developing an accurate assessment of a situation. Level $3 \mathrm{SA}$ (projection) is the ability to forecast future situational events and dynamics, aiding in timely decision making and planning. If drivers are able to successfully perceive, process, and attach an appropriate meaning to each environmental cue they perceive, then those drivers can correctly plan their response to the driving situation. With older drivers who achieve Level 3 SA, the issue then becomes one of whether or not they have sufficient time to react or execute their plan.

Current research by DeRamus et al. (2004) suggests that, indeed, if an older adult has a good cognitive assessment of a situation, is aware of the important cues / elements, has processed them correctly, and has sufficient time to plan and react, his choices tend to be as good if not superior to those of younger experienced drivers. Many of the driving scenarios in DeRamus' 
work involved non-turning situations where important cues were directly in front of the driver. This supports published data describing the types of accidents that older drivers tend to have. According to Ryan et al. (1998), older driver crash rates involving head-on, rear-end collisions and single car accidents are indistinguishable from those of drivers in the 35 to 65 age range.

However, older drivers are over-represented in angled-impact crashes (Ryan et al., 1998; Bryer, 2000). Ryan reported that in Australia, $54.5 \%$ of crashes involving drivers 80 years old or older involved an angled impact, compared with only $31.6 \%$ for drivers 45 to 49 . In the context of SA, given an angled impact crash, critical driving cues needed to avoid the collision would most likely emerge in the periphery of a driver's vision. It is well documented that older adults typically have a diminished ability to perceive and process information peripherally (Ball et al., 1988; Ball et al., 1993). Older adults also may not look to the sides as quickly or as often while driving due to age related decreases in flexibility and mobility of the neck, arms, and torso (Eby, 1998). When you combine these two factors, the result is that older drivers are subsequently less able than younger and middle-aged adults to detect and process critical cues in their periphery necessary to achieve Level 3 SA while driving.

The primary focus of the research reported here was to investigate whether or not older drivers, with no training prior to the driving trials, would incorporate compensatory behaviors to gather information in situations where critical cues were peripherally located. As a baseline, older drivers were to be compared against a cohort of younger, experienced drivers between the ages of 25 and 55. Desired behaviors consisted of turning the head or torso to monitor for oncoming traffic in a timely fashion, taking a secondary look in areas where traffic might emerge once the driver began to move into an intersection, monitoring those areas where objects or terrain features might obscure oncoming vehicles, and getting out of the intersection in an amount of time that was appropriate to the situation. We also wanted to determine whether or not drivers would indicate a change in their perception of their driving habits based upon post-drive feedback and whether or not they planned to change their real-world behavior based upon that feedback.

\section{EXPERIMENT}

Ten scenarios were constructed for the University of Massachusetts Amherst fixed-base simulator located in the Human Performance Laboratory. The simulator is a modified 1995 Saturn sedan in which the drivers could operate controls similar to any production vehicle. A virtual world was projected onto three screens in front of the car and wrapped around the vehicle subtending 135 degrees of visual angle. An eye tracker and head tracker were used throughout the drive to maintain a record of where drivers were fixating at any given point in time. Drivers were asked to follow a lead vehicle and maintain the same lane as the lead vehicle whenever possible. Scenarios were presented in two blocks of three and one block of four.

\section{Method}

Participants. Participants consisted of 18 drivers over age 70 (older) and 18 drivers between the ages of 25 and 55 (younger). The range for the younger cohort was chosen to be 25 to 55 because, in most studies, the accident rate per VMT had leveled out and reached steady state within that range. All participants were licensed drivers who had at least 10 years of driving 
experience. Participants were recruited from the student population, faculty and staff from the University of Massachusetts and from the local Amherst, Massachusetts, area.

Simulator Scenarios. Ten simulator scenarios were designed representing situations where angled impact crashes were likely to occur if an error was made. The scenarios were based upon actual local situations in which, we judged, older drivers would be more likely to fail than younger drivers. Most of these scenarios involved turns at intersections. The scenarios could be grouped into the following categories: (1) those that evaluated right turns, (2) those that evaluated left turns across traffic, (3) those that evaluated the driver's ability to detect peripheral cues, and (4) lane-change scenarios. For more detailed descriptions of the scenarios, see Romoser et al. (2005).

Experimental Design. The ten scenarios were randomly assigned to one of three blocks. Because of the odd number, block 1 contained three, block 2 contained three, and block 3 contained four scenarios. To eliminate learning effects, the order in which participants drove the blocks was randomized.

Procedure. When arriving in the lab, each participant was asked to complete a pre-drive questionnaire. The questionnaire collected demographic information, information about general vision and health, and fifteen questions about driving habits and behaviors. Five of these questions asked participants about how often they looked right and left at intersections, how often they took a secondary look towards oncoming traffic when beginning a turn, how often they increased their speed accordingly if they perceived there to be little time available to turn, how often they looked to the far right and left when approaching a crosswalks, and how often they glanced into the target lane when changing lanes on the interstate. The participant rated whether they did these things consistently, often, occasionally, or rarely/never.

After two practice drives to learn how to handle the simulator in the virtual environment, participants were fitted and calibrated with the car's ASL 5001 eye tracker. The participants drove the three experimental blocks in a randomized order. Each drive was recorded on video with the participant's eye fixations overlaid upon the video. During the drive, the experiment administrator rated the driver's handling as "acceptable" or "unacceptable" (coded as a "red flag"). A participant received a red flag for a scenario if the participant (1) failed to execute a primary look to the right or left to assess traffic in the intersection, (2) failed to execute a secondary look when proceeding into the intersection to assess oncoming traffic in areas where traffic may emerge while in the intersection, (3) took too long to turn given the time available, (4) failed to fixate and / or react to a critical target moving in the periphery, (5) merged an unsafe distance in front of another vehicle or, (6) had a collision of any kind or other unanticipated reckless action taken by the participant. After the experimental drive, the participants sat down with the experiment administrator to receive post-drive feedback. The participant's drive was replayed on a large-screen television. All ten scenarios were replayed. However, after each scenario where a red flag was received, the video was paused and the administrator discussed with the participant what went wrong in the scenario, why it could have led to a collision, and suggested compensatory strategies (such as taking secondary looks, or waiting for vehicles to move for a clear line of sight, etc.) the participant could incorporate into his or her on-the-road driving behavior to help avoid missing peripheral cues in similar situations to help avoid collisions. 
After the post-drive feedback session, the participants were provided with a post-drive questionnaire, which contained questions similar to those on the pre-drive questionnaire. The intent was to determine whether or not the participant's attitudes had changed regarding their driving habits after they had received an opportunity to view their own driving performance in the simulator replayed for them. However, the questions were slightly rephrased to capture the extent to which the participant would change his or her behavior.

Finally, in order to determine how well older drivers were able to incorporate the feedback they received into their actual driving strategy, five to six months after their session, ten drivers were invited back to the lab to drive again. In the virtual environment, scenery was changed significantly to prevent the drivers from recognizing the scenarios. Five of the drivers invited back had received the fewest red flags (low RF) and five of the drivers had received the most red flags (high RF). No driver in the experiment had previously received more than six red flags out of a possible ten. The participants were once again fitted with the eye tracker and drove all three blocks of scenarios. Red flags were determined the same way as in the first session.

\section{Results}

On average, older drivers were more than three times more likely to receive red flags as younger drivers. Older drivers received the most red flags (10 total) in the Left Turn with a 3-Second Reveal scenario. The scenario with the second most red flags was the Interstate Lane Change scenario (8) followed by the Impatient Motorcyclist and Left Turn with Hidden Oncoming Traffic scenario ( 7 each). Figure 1 summarizes the number of red flags per scenario for both older and younger drivers in the study.

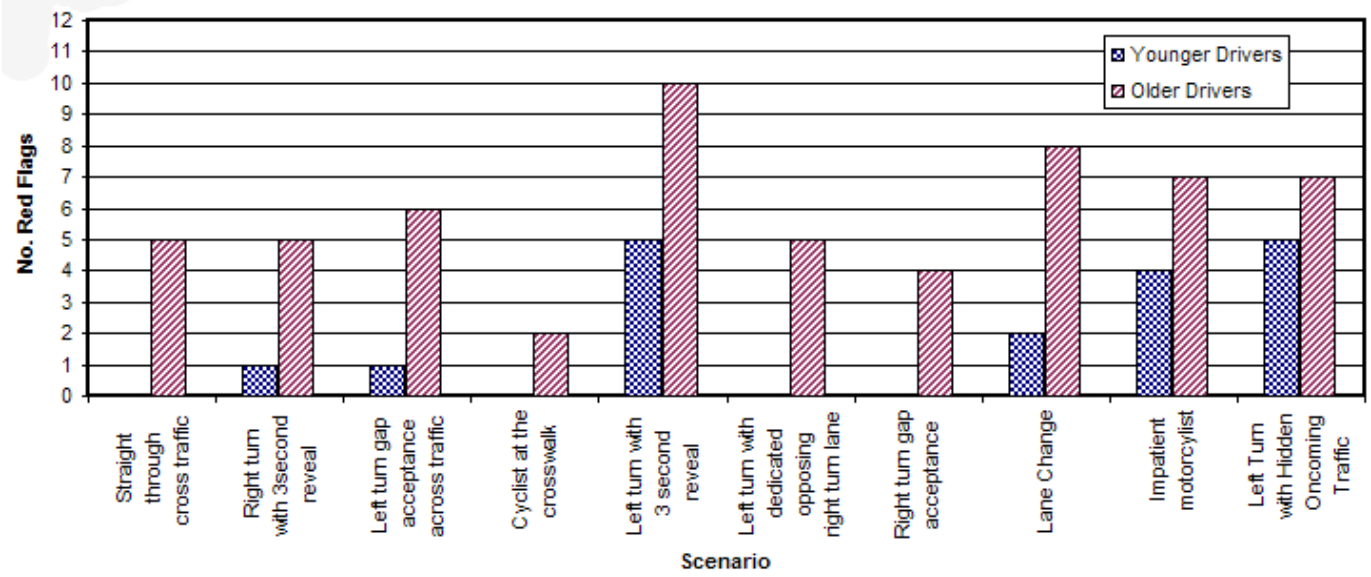

Figure 1. Red flags (errors) per scenario

The primary reasons recorded for red flags across all older drivers are summarized in Table 1. In scenarios where red flags were given, older adults on average said that they were somewhere between likely and very likely to change their driving behavior based upon the feedback that they received. Figure 2 shows that drivers from both cohorts who received feedback were generally receptive to the idea of incorporating the compensatory behaviors provided during the feedback sessions into their driving habits. 
Table 1. Frequency distribution of red flags

\begin{tabular}{|c|c|}
\hline Reason for Red Flag & Number of Red Flags \\
\hline Driver failed to look before or during a turn & 32 \\
\hline Driver turned too slowly & 10 \\
\hline $\begin{array}{c}\text { Driver merged too close to an adjacent } \\
\text { vehicle }\end{array}$ & 3 \\
\hline $\begin{array}{c}\text { Driver failed to glance into adjacent lane } \\
\text { before merging into it }\end{array}$ & 2 \\
\hline $\begin{array}{c}\text { Driver failed to fixate on the risk in the } \\
\text { periphery field of vision }\end{array}$ & \\
\hline
\end{tabular}

Of those participants who were invited back for a second driving session, both the low RF and high RF drivers on average experienced a reduction in red flags. Low RF drivers experienced a $12.5 \%$ reduction in red flags on average. High RF drivers showed even greater improvement with an average reduction of $20.8 \%$. Anecdotally, in an informal interview after the second session, several participants reported that after their feedback session, they had made a deliberate effort to try and incorporate the strategies they were given into their day-to-day driving.

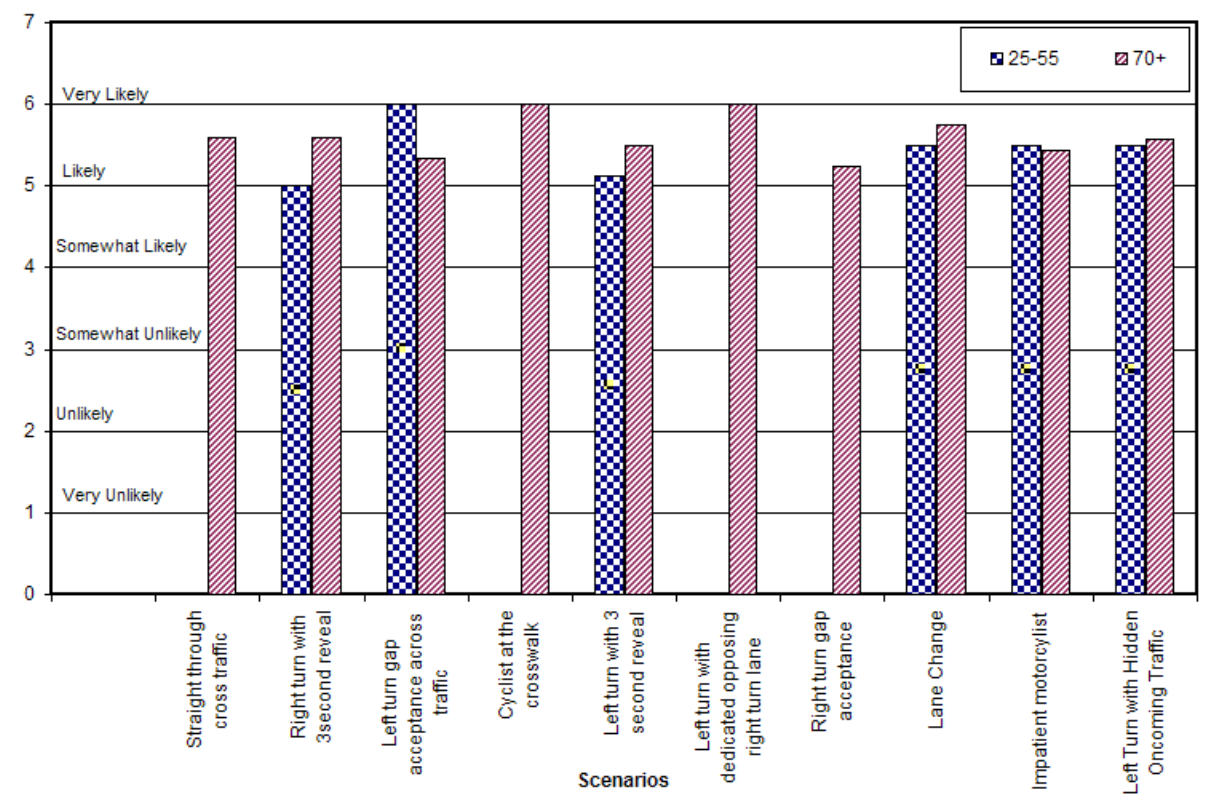

Figure 2. Driver willingness to change driving habits when red flagged

\section{CONCLUSIONS}

Results such as ours demonstrate that simulation-based training can also be an effective means of instructing drivers. Having drivers drive through a series of virtual scenarios and then replaying their actual drive back for them provides a high degree of face-validity and really solidifies, in the driver's mind, the need to make adjustments to his or her driving behaviors. Our drivers demonstrated a genuine interest in their results and were often surprised when our findings did not exactly match what they thought of themselves as drivers. When feedback was provided, both older and younger drivers reported that they would likely change their driving behavior. 
Our results also demonstrate that post-drive feedback can also be an effective means of increasing older drivers' overall situational awareness. Most of the feedback the older drivers received involved taking more primary and secondary looks toward oncoming traffic when in an intersection. This extra head-turning activity allows the older driver to collect more information about their environment and makes it more likely that they will achieve accurate Level 3 SA.

\section{REFERENCES}

Ball, K., Beard, B. L., Roenker, D. L., Miller, R. L., \& Griggs, D. S. (1988). Age and visual search: Expanding the useful field of view. Journal of the Optical Society of America, A., 5: 2210-2219.

Ball, K., \& Owsley, C. (2000). Increasing mobility and reducing accidents of older drivers. In: Schaie, K. W., \& Pietrucha, M. (Eds.), Mobility and Transportation in the Elderly. New York, NY: Springer Publishing Company, Inc.: 213-250.

Ball, K., Owsley, C., Sloane, M. E. Roenker, D. L., \& Bruni, J. R. (1993). Visual attention problems as a predictor of vehicle crashes in older drivers. Investigative Ophthalmology \& Visual Science, 34: 3110-3123.

Bryer, T. (2000). Characteristics of motor vehicle crashes related to aging. In: Schaie, K. W., \& Pietrucha, M. (Eds.), Mobility and Transportation in the Elderly. New York, NY: Springer Publishing Company, Inc.: 157-206.

Cook, L. J., Knight, S., Olson, L. M., Nechodom, P. J., \& Dean, J. M. (2000). Motor vehicle crash characteristics and medical outcomes among older drivers in Utah, 1992-1995. Annals of Emergency Medicine, 35: 585-591.

De Raedt, R. \& Ponjaert-Kristoffersen, I. (2000). The relationship between cognitive / neuropsychological factors and car driving performance in older adults. Journal of the American Geriatrics Society, 48: 585-591.

De Ramus, R., \& Fisher, D. L. (2004). The effect of driver age and experience on risk assessment and risk prediction. In: HFES 2004: Proceedings of the Human Factors \& Ergonomics Society, 48 ${ }^{\text {th }}$ Annual Meeting: 2627-2631.

Eby, D. W., Trombley, D., Molnar, L. J., \& Shope, J. T. (1998). The assessment of older driver's capabilities: A review of the literature (Report No. UMTRI-98-24). Ann Arbor, MI: University of Michigan Transportation Research Institute.

Endsley. (2000). Theoretical underpinnings of situation awareness: A critical review. In: Endsley, M. R., \& Garland, D. J. (Eds.), Situation Awareness Analysis and Measurement. Mahwah, NJ: Lawence Erlbaum: 3-32.

Evans, L. (1987). Fatal and severe crash involvement versus driver age and sex. In: $31^{\text {st }}$ Conference of the American Association of Automotive Medicine, New Orleans: 59-78.

Fozard, J. L. (2000). Sensory and cognitive changes with age. In: Schaie, K. W., \& Pietrucha, M. (Eds.), Mobility and Transportation in the Elderly. New York, NY: Springer Publishing Company, Inc.: 1-44.

Graca, J. L. (1986). Driving and aging. Clinics in Geriatric Medicine, 2: 577-589. 
Hakamies-Blomqvist, L. (1996). Research on older drivers: A review. IATSS Research, 20: 91101.

Hakamies-Blomqvist, L., \& Wahlstrom, B. (1998). Why do older drivers give up driving? Accident Analysis and Prevention, 30: 305-312.

Klavora, P., \& Heslegrave, R. J. (2002). Senior drivers: An overview of problems and intervention strategies. Journal of Aging and Physical Activity, 10: 322-335.

Lyman, S., Ferguson, S. A., Braver, E. R., \& Williams, A. F. (2002). Older driver involvements in police reported crashes and fatal crashes: Trends and projections. Injury Prevention, 8: 116-120.

Romoser, M., Fisher, D. L., Mourant, R., Wachtel, J., Sizov, K., Kennedy, S. I., \& Andra, M. (In press). Use of a drving simulator to assess older adults' critical driving skills. Transportation Research Record.

Ryan, G. A., Legge, M., \& Roseman, D. (1998). Age related changes in driver's crash risk and crash type. Accident Analysis and Prevention, 30: 379-387. 